\title{
EAl Endorsed Transactions

\section{ANFIS based hybrid approach identifying correlation between decision making and online social networks}

\author{
Areeba Rahman ${ }^{1}$, Nageen Saleem ${ }^{1}$, Aysha Shabbir ${ }^{1}$, Maryam Shabbir ${ }^{1}$, Muhammad Rizwan ${ }^{1, *}$, Shahid \\ Naseem $^{2}$, Fahad Ahmad ${ }^{1}$ \\ ${ }^{1}$ Department of Computer Sciences, Kinnaird College for Women, Lahore, Pakistan \\ ${ }^{2}$ Department of Information Sciences, University of Education, Lahore, Pakistan
}

\section{Abstract}

The fast-growing use of online social networks (OSNs) has prompted stakeholders to change their market strategies and hence
have raised several questions on the users' decision making (DM). OSNs, as being regularly used, have resulted in playing a
significant role in supporting consumer's rational DM. We use a hybrid approach i.e. an online survey and fuzzy model
development to indicate how OSNs have resulted in giving an impact on the decision making. The research depicts that OSNs
support and empower users in the DM process specifically in Rationality, Design, \& Choice and the model predicts the DM
strategy of each user according to their present decisions using fuzzy logic. Our results also reveal that different types of users
(observers, seekers, and advisers) have significantly different participation styles, which in turn have an impact on the efficacy
of the DM process. We discussed the implications for OSN designers and developers based on the findings from the research.

Keywords: Rationality, Decision Making, Online Social Networks, Fuzzy Logic, Impulsive Buying.

Received on 24 March 2020, accepted on 11 July 2020, published on 22 July 2020

Copyright (C) 2020 Areeba Rahman et al., licensed to EAI. This is an open access article distributed under the terms of the Creative Commons Attribution license, which permits unlimited use, distribution and reproduction in any medium so long as the original work is properly cited.

doi: 10.4108/eai.13-7-2018.165669

*Corresponding author. Email: muhammad.rizwan@kinnaird.edu.pk

\section{Introduction}

Decision making marks its roots from the genesis of humans as life is all the sum of one's choices, this process has had been evolved, altered, and explored in various forms to explain and relate it to modern-day scenarios. Hence it is a composite research field depending upon various physiological aspects of an individual.

In the modern era face to face interactions and communications are being rapidly replaced by one click away from social connections over different networks [1]. A social network is a social composition providing a means of interaction between various actors i.e. organizations and individuals sharing some common interest [2]. Online social networks, therefore, have become an easy way of exploring world events, improve information discovery, give the ongoing trends, and allowing the user to do selective perception. Most online social sites share some common aspects, giving the user the feature to create a profile, update their information, add people of their interest, like and comment on the material they see [3], compare their lives, know the trend of clothing, styling and decide what they desire for themselves. This research focuses on analyzing the process of DM using OSNs.

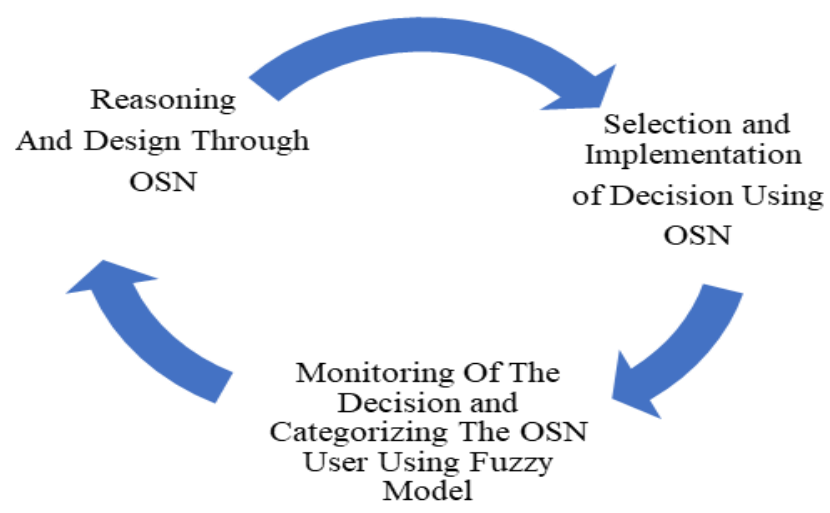

Figure 1. DM process explained 
Generally, DM is a simple art of selecting from more than one available possibilities which can also become a complex task of solving a complicated problem by offering solutions through intuition or reasoning or both. As it is known that the process of DM comprises various stages (Reasoning,
Design, Selection, Implementation, and Monitoring) and the user has unique ways of decision making from rational to spontaneous. Thus, OSNs play a significant role in letting the user decide what type of decision-maker he/she is

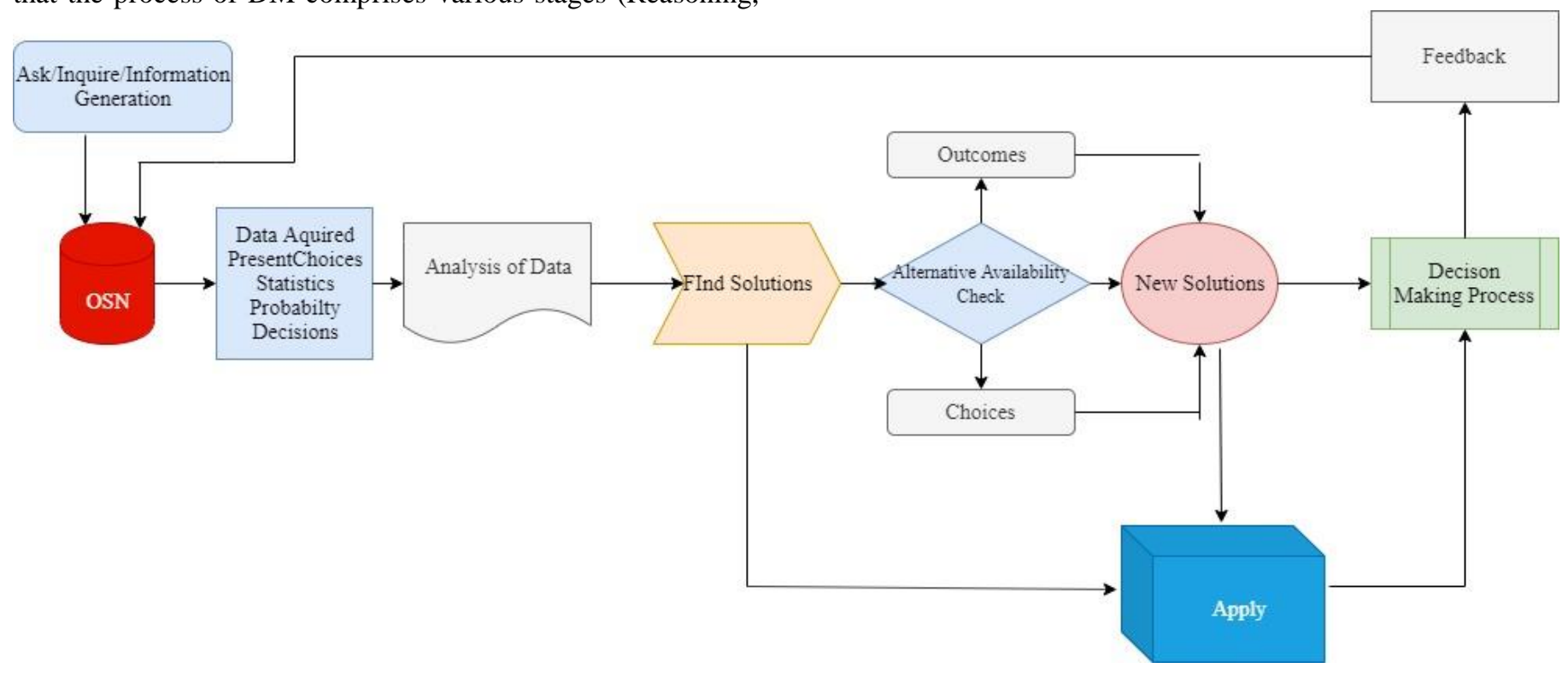

Figure 2. OSN architectural framework support decision-making process

Meant to be and will be in the future. Fuzzy systems, therefore, provide an opportunity to make a rule base which gives the value of 'truthiness' [4]. In this case, it provides the prediction that the user will fall in which category intuitive, rational, independent, avoidant, observer, the advice seeker, or independent [5]. The decision phases in OSN is described in Figure 1. The whole process of Decision making within the OSN is depicted in Figure 2. It states how decision making is presently done with the help of OSNs.

As the data is generated by the user is isolated by the OSN based on the information. It then analyses data and finds the available solutions and adds new solutions too. Feedback is then provided to the OSNs to work for the future. Our study is based on proving the positive impact of OSNs on decision making and providing the categories by fuzzy neural systems of the user based on his decisions. This paper will focus on the impact of OSNs on consumer decision making. It will help various stakeholders to design their advertisement accordingly based on the category of user and his predicted future behavior using OSNs platforms.

In this research, the rest of the paper is organized as follows. We discuss background and related necessary literature in Section II. In section III, the data being gathered from online surveys, interviews by netnography is analyzed and normalized. In Section IV, the developed hypotheses are tested defining the problem statement of the research. In Section V, we explain our proposed architecture and implementation of the architecture. Finally, section VI, concludes the research explaining the future aspects.

\section{Background and related literature}

\section{$2.1 \mathrm{OSNS}$}

Online social networks have gained rapid importance during these recent years other users have become an audience for one user [6]. Their clothing, styling, study, research has become a source of information for others. People tend to make important decisions by comparing their lives with others. In one's subconscious, he creates an image in the mind of his outlook if he opts for a certain attire which is liked by many. Thus, unintentionally he decides to choose a dress, shoes, or any object more liked by others or gives value to what others like. Thus, he tends to become one of the consumers which fall in the categories discussed in section I. OSNs, therefore, have become a platform that creates an impact direct or indirect on the user. These platforms have attracted many to advertise their products [7]. They have evolved from casual chatting sites to specific user-oriented sites [8]. These have undergone various updates every month to reach the expectations of users and compete appropriately in the modern technology era. There have had been categories like Facebook a social interactive site, 
twitter famous for tweets, Instagram a picture-based social site, youtube a site based on videos.

All these sites are now evolving as the user when he likes something he generates a data, the user as according to the legal policy of these sites compromises on his privacy as this data is being used by the developers [9]. This generated data assist them to isolate the information based on the class, likes, region, interest of the user. Thus the topic analysis is done using the information provided by the user, the creation of a topic based on the analysis, and the change of topic or need of use over time [10].

\subsection{Decision making}

Decision making is a vast study which is influenced by cognition of an individual or a system. This process revolves around two major categories i.e. rational decision making where solutions are derived from a known environment and the irrational decision making where the decisions are predicted due to the uncertain environment.

There have been several decision-making models that have been extended evolved and altered according to need and time. [15]. Simon's model is the most promising and recognizable in the proposed models [14][16]. Various extensions and models have been evolved which then included stages of DM process i.e. monitoring and implementation.

\subsection{Fuzzy systems}

Fuzzy systems provide a framework [10] of mapping human attributes to a mathematical framework. It determines the degree of truthness. The numerical data is mapped via a nonlinear universal approximator, an attribute of neural network. The fuzzy basis function is expansion is generally considered very powerful and appropriate for reasoning as its basic function can be derived from numerical data or linguistic knowledge, both of which can be drawn into if-then rules. They are nowadays used for making certain logic control systems to measure the performance [17]. They have been used over a long time for the development of certain adaptive systems [9].

\subsection{Problem statement}

OSN are significant wellsprings of online communications and substance sharing, subjectivity [2], appraisals, approaches, assessment, impacts, perceptions, emotions, conclusions and estimations articulations borne out in-text, conversations, audits, web journals, comments, news, responses, or some different archives. Based on the literature review, we scrutinized various researches that provided a theoretical framework of how OSN affects the decision-making process of the user using different tools. In various researches, there lied a need for a model that could take certain variables and process this information in a more appropriate way of detecting the user category. The researchers used SPSS tools or similar to detect the correlation. However, a system that could isolate the type of decision-maker was not directly seen. Certain hypotheses were developed and tested. The data was not Labeled, normalized, and applied over a mathematical model to generate a solution. Thus the need to get to know the category of the user is necessary because it will help various stakeholders to target their advertisement according to the behavior of the user. Hence, a system that could predict the user's current and future behavior could give an edge to OSNs as well to improve their design. The current process of decision making could be improved if a certain framework existed.

\subsection{Motivation}

OSNs are a term used to depict online administrations that inside a domain permit people to make a public/private profile, with the end goal that inside the network they can openly communicate with different clients. Lately, informal community research has been done utilizing information gathered from online collaborations and unequivocal relationship interfaces in OSNs (e.g., Instant Messenger, Flickr, LinkedIn, Facebook, and so forth). The capacity to gather this sort of information by mechanical methods has suggested a noteworthy move in the research domain of social networks, prompting the development of another computational social science. The quickly exceeding utilization of OSNs has incited partners to change their market systems and consequently have brought up a few issues on the clients' DM. OSNs, as being normally utilized, have brought about assuming a noteworthy job in supporting shopper's rational DM. A hybrid approach is utilized, a fuzzy model with an online survey to show how OSNs have brought about giving an effect on the DM process. The manuscript delineates that and supports clients in the DM procedure explicitly in Choice, Design, and Rationality and the model predicts the DM technique of every client as indicated by their current choices utilizing fuzzy logic. This research additionally uncovers that various kinds of clients (advisors, seekers, and observers) have fundamentally unique styles of participation, which thus affect DM procedure effectiveness.

\section{Literature review}

Decision Making is a speculative and real-world idea that is dependent on the intellectual perception of the one who is making the decision. The procedure of decision making can be structural and chaotic. With the evolution of the internet the same sites including many others, have come to a noteworthy segment of Internet users that are producing the highest engagement rate between users. Some online social networks are audience-based while some are growing globally.

For example, Youtube, Google plus these are the sites that are accessed by the whole internet world while some social networks are designed for a specific community. On the usage of these social networks, people make their decisions. One of the main reasons is that these networks have their influence on the users. There are millions of social networks that are

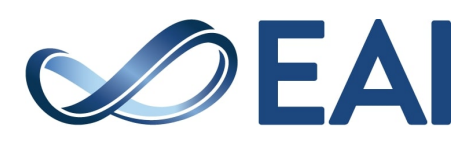


designed for different purposes and they are playing a very vital role in supporting the decision making the process for many people worldwide. Many researchers analyzed the impact of online social networks on decision making in various ways. Some of them have been discussed in the following sections.

Valeri Sadovykh and David Sundaram used the method of online surveys to evaluate how much support online social networks to provide the decision-making process. There results and analyses showed that online social networks allow and help users in decision making. The results also exposed that the type of user (audience) has a great impact on the decision making process and its efficiency [11]. David Sundaram et al. proposed a solution of decision making through online social networks by a pluralistic approach that includes qualitative and quantitative research using netnography technique. They found out that OSN does or does not influence decision making [12]. Ghania Bilal et al. analyzed the decision-making process through social media by specifying the decision-maker. which depends on the need of people before deciding OSN [7]. Yan Jia et al. researched on Topic analysis for social online networks. The research summarizes the common factors and features of the current topic analysis model that covers the effect of community, users, and influence on topic analysis [10].

Erkan Akar et al. researched on the specific community of decision-makers. They examined those people who follow sports and analyzed the effect of their decisionmaking process via Facebook. They used SPSS 20 and AMOS 21.0 tools for their analysis and proposed that Facebook has a great impact on users [13]. Various decision models and reviews are explained by R. Azuma[15]. The process of decision making is not new, its roots back to cognition and behavior as the theories of decision making and its traditional model have been discussed by H.A Simon [14].

Gulsum Akuzu et al. proposed a fuzzy logic-based model to handle the privacy issues on the data that is shared through social networks by using the data sensitivity value and confidence value in fuzzy systems [15]. The use of fuzzy in developing adaptive models i.e performance measurement of systems is discussed Darwin O. et al [16]. Ralph and Alasandro worked on the behavior of 4000 students and evaluate the patterns of information revelation in online social networks [1]. Mohsin Jameli et al. proposed a comparative analysis of social networks in various aspects. A detailed framework of OSNs is given in as well [2]. A direct description of fuzzy systems is discussed by MM Gupta et al. It describes the fuzzy logic background and how it has had been used [4].

Liu Qingy et al. worked on a theoretical model on the impulsive buying behavior of the consumer, how people tend to buy without the need of buying and most of the people end up buying the unneeded stuff [5]. The change in human behavior using Online Social networks is analyzed by Jushua $\mathbf{J}$ et al. described how these social sites e.g. Facebook, Orkut, Google etc draw a relationship with decision making [6]. QiFei Yan et al similarly depict how the social networks have evolved using the Clique-superposition model and the model generated patterns old and new [8]. The use of OSNs has also created a privacy loophole for its users as discussed by Nobubele et al. considering the legal policy of various social sites [9].

Fuzzy Petri Net (FPN) is received as the knowledgemodel of operators. In the manuscript, the specialists can communicate and interact with one another to share data, scrutinize, and check each other's information, and cooperatively make determination dependent on numerous specialists' information through the process of argumentation [17-18]. OSN has been created as an extraordinary point for its clients to speak with their intrigued companions and offer their suppositions and thoughts, photographs, and recordings reflecting their states of mind, emotions, and notions. This makes a chance to examine the OSN data for client's emotions and notions to analyze their states of mind and mentalities when they are conveying utilizing these online tools. In the manuscript [19], the author intends to perform an analysis of depression on Facebook information gathered from an online open source. To examine the impact of depression recognition, the author proposed a machine learning strategy as a scalable and efficient technique.

Various uses of Fuzzy Cognitive (FC) Maps utilizes static causal connections to provide the connection among various ideas. But, a causal effect may produce results quickly or combined over a timeframe. By considering two FC models with an equivalent causal structure, state-set, decision-function, and causal linkage qualities, in case their causal connections have various elements, they can have altogether distinct or even entirely different hidden-patterns. This research [20] proposed a simple to utilize model to elucidate the causal connection's dynamics in FC maps. Moreover, the transformation among FC models is also feasible [21].

\section{Data analysis}

The main objective of this survey is to depict how OSNs support decision making and to gather the data to analyze various kinds of decision-makers within the consumers. This study was conducted using Google Forms as the platform and the data collected is considered as responses. The data generated shows how people rely on social media platforms when deciding something. It also depicts how OSNs have become an important part of our lives and have attributed to affect the rationality of our choices. The reason that various markets have a huge consumer base especially in countries like Pakistan besides being on low finance is impulsive buying and brand consciousness of the user.

This comparison based life has harnessed the rationality in decision making. The data was normalized by classifying the data into ordinal and nominal forms. 146 respondents took part in the survey, all belonging from the different backgrounds using social media platforms. The data being asymmetrically skewed as $71.7 \%$ of the respondents were female while $28.3 \%$ were male $47.6 \%$ belonged to the age group of 16 - 23 while 44.8 percent belonged to $23-29$, only 7.6 percent were $30-40$, and no one from 45 and above. $29.5 \%$ completely agreed that their choice depends upon the number of likes being assigned to specific clothing on Facebook, $39 \%$ 
went with sometimes while 31.5 percent disagreed. $52.1 \%$ agreed that OSNs usually prompted them for impulsive buying, $26 \%$ completely agreed while 23.3 percent disagreed that they go for impulsive buying.

On the question of what do they think that OSNs are affecting our rationality of choices, 56.2 percent agreed completely while 43.8 percent disagreed.

On the question based on that would they go with the trend or marginalize with their celebrity choices and statements when using twitter 102 out of 146 responded, out of which 30 said that they don't use twitter, 45 agreed that they follow their celebrity and 35 went with the trend while remaining relied on their own opinion. However, when they were asked if they desired to buy a dress what would they do order online or go to the outlet, $60.7 \%$ responded they will take the ideas online and buy the dress from the outlet while $33.8 \%$ said they will search online for what they are looking for.

On the query that do they think that social media affects our rational choice or is helpful, 112 people responded, $50 \%$ agreed to the helpful option while 45 percent said them to be a source of confusion and affecting our rational choice. 53.1 $\%$ agreed that on the question that they collected tweets and images from OSNs to use it in the future. On the inquiry that which platform of social media they would prefer the most if they wished to make an opinion, $39.2 \%$ went for Instagram, $24.5 \%$ with Facebook,33.6\% went with online sites while the rest with twitter. The results of a few research questions from the survey are displayed in Figures 3, 4, and 5.

Does Facebook or Instagram or the sales you see on brands prompt you for impulsive buying?

146 responses

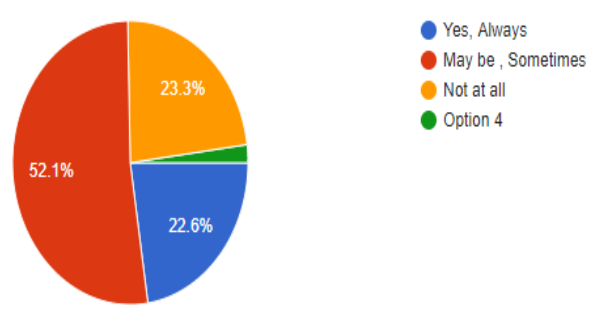

Figure 3. Impulsive buying behavior of the consumer

Do you collect tweets, pictures of things you like on OSNs to use it in the future? 143 responses

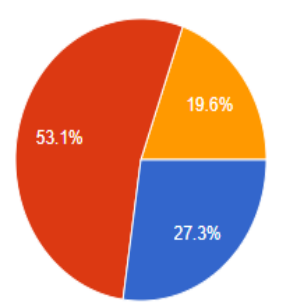

Yes, always

Yes, sometimes

Not at all

Figure 4. Response from an online survey
If you want to buy a new dress for a function, What would you do?

145 responses

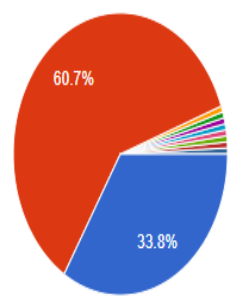

- Search various brands and outfits on...

Go to the outlet and buy what you thin. - Search brands but in the end prefer to.

- I eiefer brand i will go to brand and buy

I'll look online just for inpso, then get it.

- Go to the mall and search

Search on the internet first then go to..

ill design it myself

$\Delta 1 / 2 \nabla$

Figure 5. Respondents answer

\section{Proposed architecture}

We propose a hybrid technique using a qualitative and modelbased study that describes the correlation between OSN and DM and detects the kind of decision-maker as shown in Figure6. One phase adds to the other phase. The phase 1 of the architecture explains the existence of a positive correlation between OSN and DM. In Phase 1 the online survey and interviews are carried out to gather information about the relationship of OSNs and Decision Making and to know the impact of OSNs on the decision-making process. The data is analyzed and divided using the Likert scale and other ordinal and nominal forms. Two basic hypotheses were built and the conclusion was drawn manually after data being analyzed.

Phase 2 of the proposed framework gets the attributes and properties by the input values from phase 1 and detects the decision behavior of the user using a fuzzy model as shown in Figure 7. The parameters are defined, categories and ranges are defined, the data is done into labels, and the rules were set and applied to the neural Mamdani model. It then categorizes him into a rational, independent, observer, avoidant, intuitive, or advice seeker. A rational decision-maker will not be affected by OSNs rather his buying will depend upon needs opinion will depend upon self-analysis and his motivation, an intuitive one will depend upon his gut feeling and mood to decide what he wishes to do the observer will only observe or may kill his time surfing various OSNs, the avoidant will try to avoid all the forms which lead him to create opinion, do impulsive buying or creates the desire, the advice seeker will always look into various platforms and choose amongst them. However, all will consider OSN as a significant platform.

Figure 7, a model for decision behavior detection has been proposed. Firstly, the inputs are identified based on online social network users. Inputs are classified as Age, Gender, Social Site, Rationality, no.of likes, Reviews, and Post Purchase Consumption. The inputs are further classified into multiple categories which have been discussed in the later section. After defining the input, the data is analyzed by a fuzzy system which is the Fuzzy Inference Mamdani model. Then a Neuro-fuzzy designed handles the data from defuzzifier and provides the crisp value. The crisp value is further analyzed and detects the decision behavior e.g. if the decision is independent or rational or intuitive.

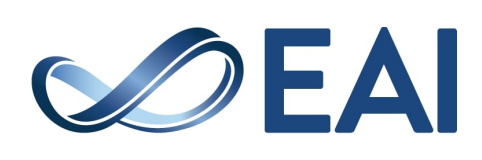




\section{List of hypothesis}

- Online Social Networks do not have an impact on consumer decision making

- The first hypothesis is given below

- Social Networks influence rational choices of the consumer

- Facebook has a positive impact on consumer decision making

- YouTube has a positive influence on consumer decision making

- Twitter has a positive influence on consumer decision making.

- Instagram has a positive influence on consumer decision making

\section{Implementation}

For the implementation purpose, we used MATLAB and fuzzy inference systems. A fuzzy system is one of the most operative and effective techniques of qualitative computers. It does not follow the standard "True or False" method. It gives the true values and uses membership functions. We specifically used the Mamdani model over Sugeno because it is intuitive and gives non-continues output surface and membership function output. It also provides MISO (multi-input single output) and MIMO (multi-input multi-output). Moreover, it is compatible with human input and has extensive acceptance.

In the Sugeno model, firstly inputs variables are defined and then the membership functions are being demarcated.

Table 1. Input \& Membership functions with Ranges

\begin{tabular}{|l|l|l|l|}
\hline \multicolumn{1}{|c|}{$\begin{array}{c}\text { Input } \\
\text { Variables }\end{array}$} & \multicolumn{1}{|c|}{$\begin{array}{c}\text { Membership } \\
\text { Fn.1 }\end{array}$} & \multicolumn{1}{|c|}{$\begin{array}{c}\text { Membership } \\
\text { Fn.2 }\end{array}$} & \multicolumn{1}{c|}{$\begin{array}{c}\text { Membership } \\
\text { Fn.3 }\end{array}$} \\
\hline Age & Teen [10-19] & Young[20-30] & Adult[30-45] \\
\hline Gender & Male[18-40] & Female[18-40] & $\ldots \ldots \ldots \ldots \ldots$ \\
\hline Rationality & Reasoning[5-15] & Design[15-30] & $\ldots \ldots \ldots \ldots \ldots$ \\
\hline No. of likes & Min[50-100] & Avg[95-200] & Max[150-500] \\
\hline Social Sites & $\begin{array}{l}\text { Facebook } \\
{[10-30]}\end{array}$ & $\begin{array}{l}\text { You Tube } \\
{[25-35]}\end{array}$ & $\begin{array}{l}\text { Instagram } \\
{[30-50]}\end{array}$ \\
\hline $\begin{array}{l}\text { Post-Purch. } \\
\text { consump }\end{array}$ & $\begin{array}{l}\text { Good Quality } \\
{[\mathbf{8 - 1 0}]}\end{array}$ & Avg[7-10] & Bad[0-5] \\
\hline
\end{tabular}




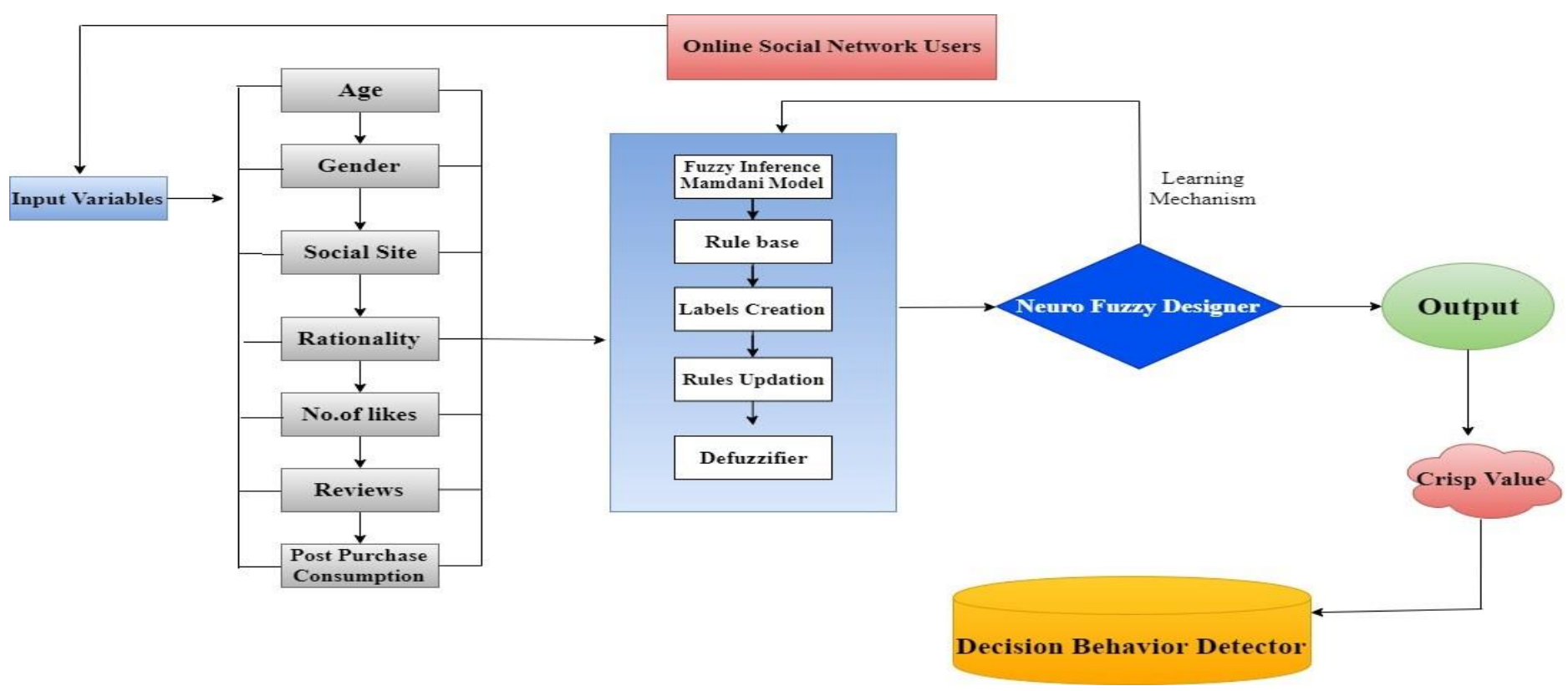

Figure 7. Proposed architecture for decision behavior detection

The following are the rules defined in the Fuzzy system Mamdani model. The rules are based on if then, some of them are listed below.

$>$ If Age is young AND gender is female AND no. of likes are average AND reviews are good then the user is Intuitive.

$>$ If Age is adult AND gender is male AND rationality is design AND no. of likes are minimum AND reviews are bad AND post-purchase consumption is average THEN user is avoidant.

$>$ If age is young AND gender is female AND social site is Instagram AND no. of likes are average AND reviews are bad THEN user is an observer.

$>$ If age is young AND gender is male AND social site is Facebook AND rationality is reasoning AND no. of likes are maximum AND reviews are good AND postpurchase consumption is good THEN user is rational.

Analyzing the user category who decide an online social network is nothing less than a challenge. There are multiple ways to do it. We proposed a solution that is the fuzzy inference system based and take inputs, then we define some membership functions and rules are made. After making rules from input and output variables rules become if then based. In the next section, we will illustrate the working of MATLAB and the fuzzy inference Sugeno model.

\subsection{Fuzzy inference system using mamdani model}

In fuzzy inference system, Sugeno model is developed, by defining age, gender, social sites, rationality, and no. of likes, reviews and post-purchase consumption as input variables as shown in Figure 8 and user category as output variable which includes rational, intuitive, independent, avoidant, observer and advice seeker.

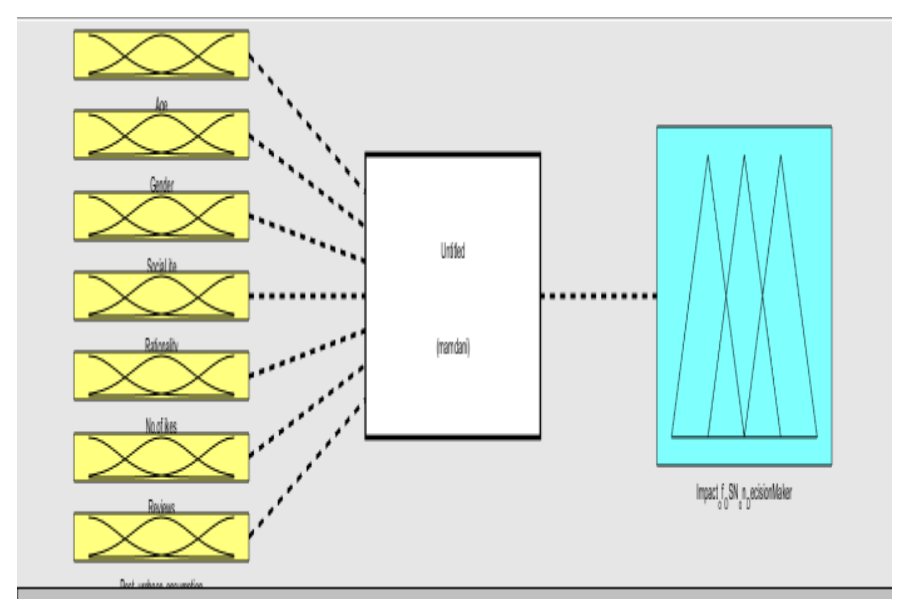

Figure 8. Fuzzy model inputs 


\subsection{Membership Function Plot}

The input is being taken and according to those the membership function along with their range has been defined e.g teen, young, adult, good, avg, bad as shown in figure 9 .

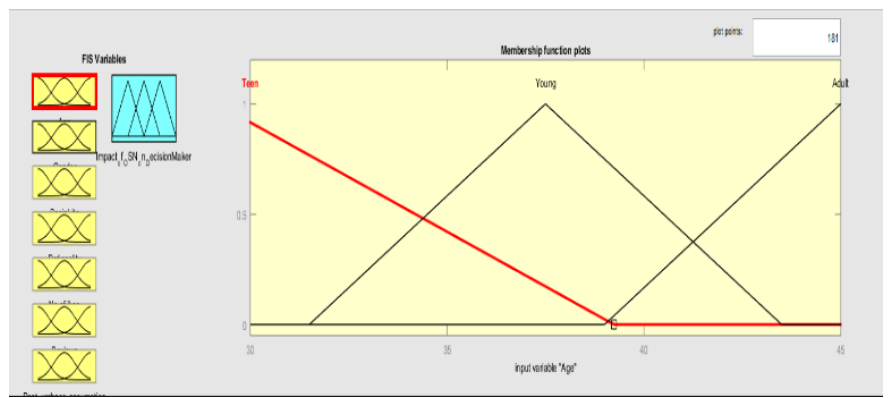

Figure 9. Membership functions defined

\subsection{Rule Viewer}

The set values are obtained and processed through rule viewer, which has if-then based rules. The rule viewer represents how certain membership functions have an impact on the final results. The rule viewer presents the live view of the fuzzy inference system process. The system has a single output with different membership categories. Every column depicts a variable and every rule is an order of plots. In the fuzzy inference system process, there are constant and linear membership functions available. Both are different and have an impact on the output variable. We used linear membership functions in our paper as shown in Figure 10. The rules are defined and then a base is formed by the system itself to check the biasness of the rule base. It tells how the defined rules are symmetric, asymmetric, or skewed.

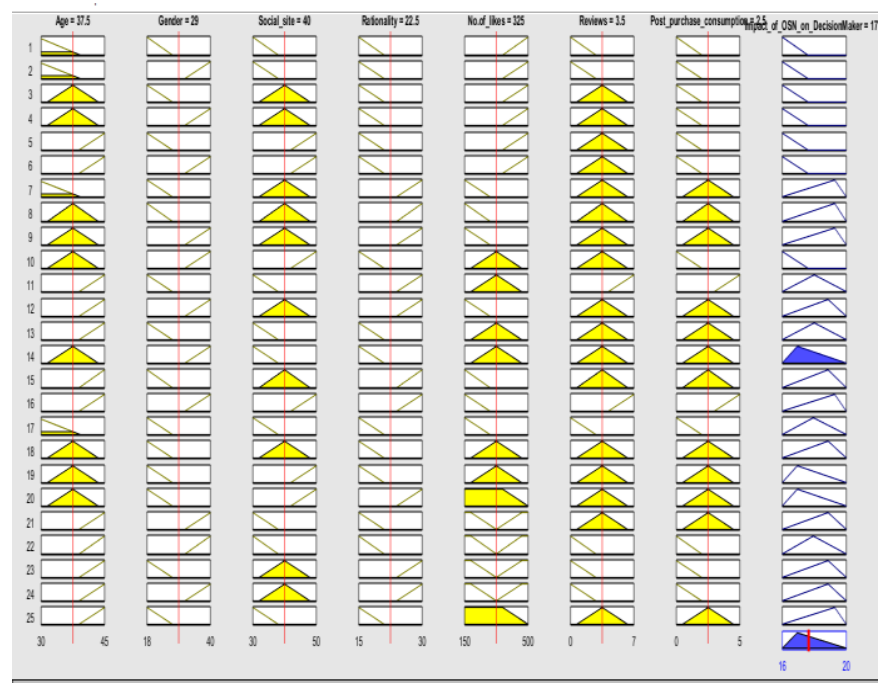

Figure 10. Rule viewer and symmetry

\subsection{ANFIS-testing and training performance}

While some data is used to generate the knowing output of the model as shown in Figure 11 and Figure 12, the other is fed to the model for training and testing purposes so that the model must be adaptive. If an advice seeker comes to the model again, the model must just by analyzing the input variables show that the user lies in the defined category. $70 \%$ of processed data is used for training purposes while $30 \%$ of the same data is used for testing as shown in the above diagram. With the resultant data and simulations, the error rate is reducing when the data is being tested and trained multiple times.

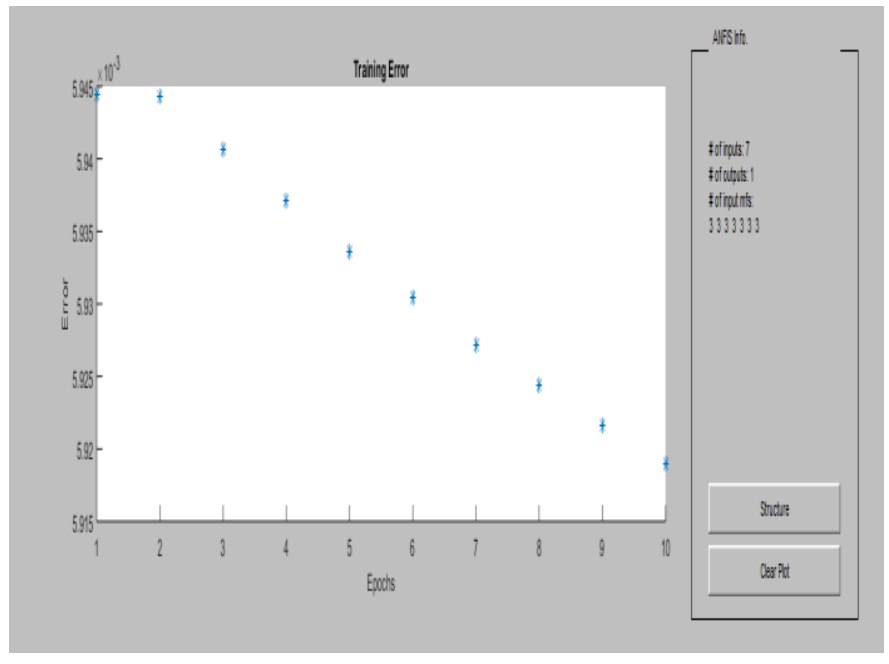

Figure 11. Training error reduced

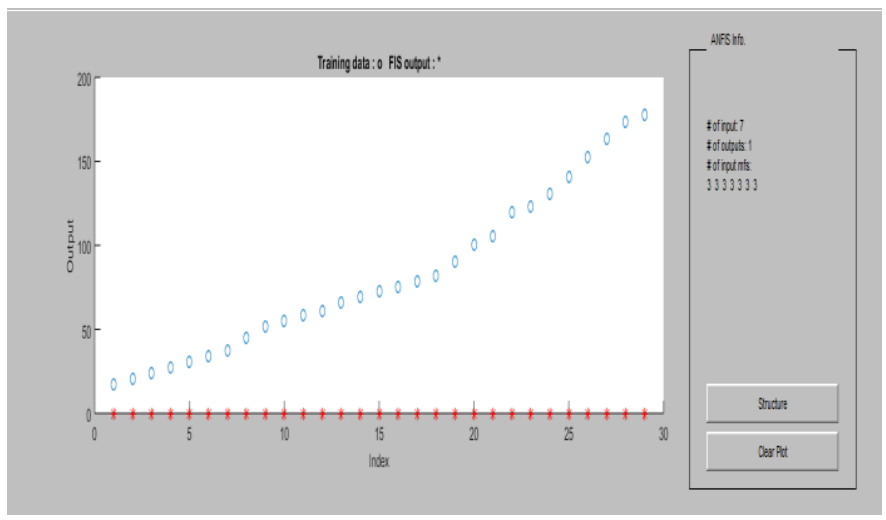

Figure 12. Dataset training

\subsection{Surface Viewer}

The two functions i.e ruleview(fis), ruleview(fileName) are called in matlab to get the surface viewer which gives the output 
of the FIS system as shown in Figure 13. 2 inputs (age and gender) have been chosen and the output is plotted against them. Each input collaborates to give the output. The six inputs form nodes and interrelate to give the kind and category of a user as shown in Figure 14. The surface viewer calls surface viewer is a three-dimensional model of the output against the input. It gives the generic results by selecting one, two, or multiple inputs and plots the output against them. In this case, 2 inputs are constant. It is one of the graphical user interface GUI tool that provides an easy view of the output.

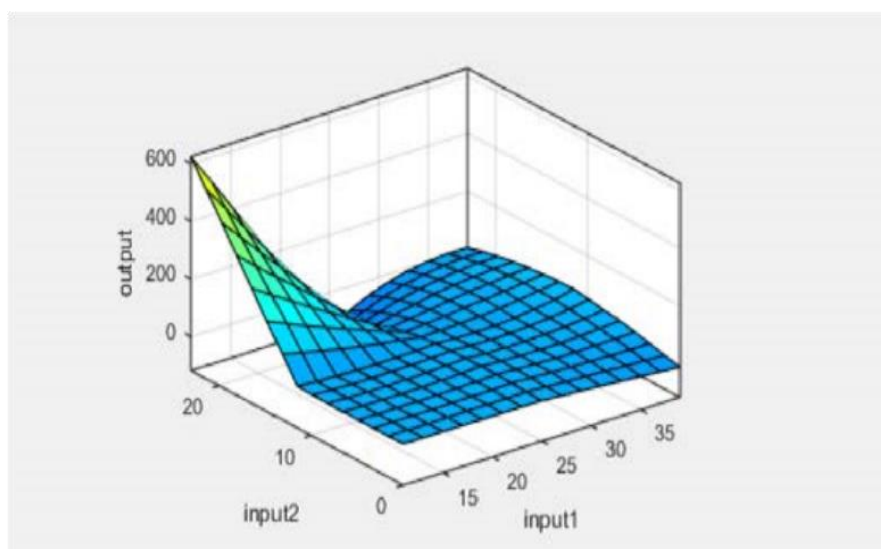

Figure 13. Surfview of output

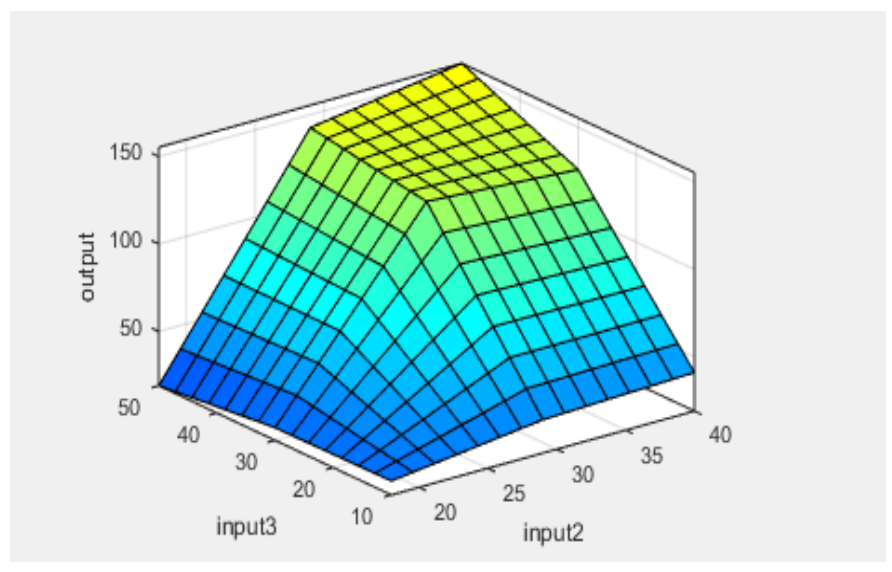

Figure 14. Surfview of output

In the above figure 14, 2 inputs Gender and Rationality have been used as input and the output shows the user category. It is seen that multiple inputs have different impacts on output. In the rest of the figures, the inputs are changed and their impact on the output is shown.

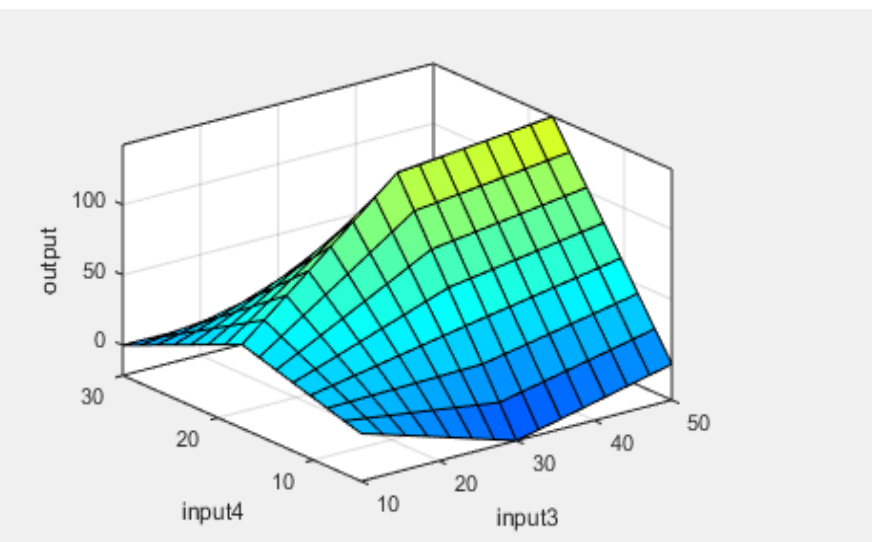

Figure 15. Surfview of output

Rationality and No. of likes are used as input variables and the user category is continuously changing. It shows that by changing the input variables the user category varies.

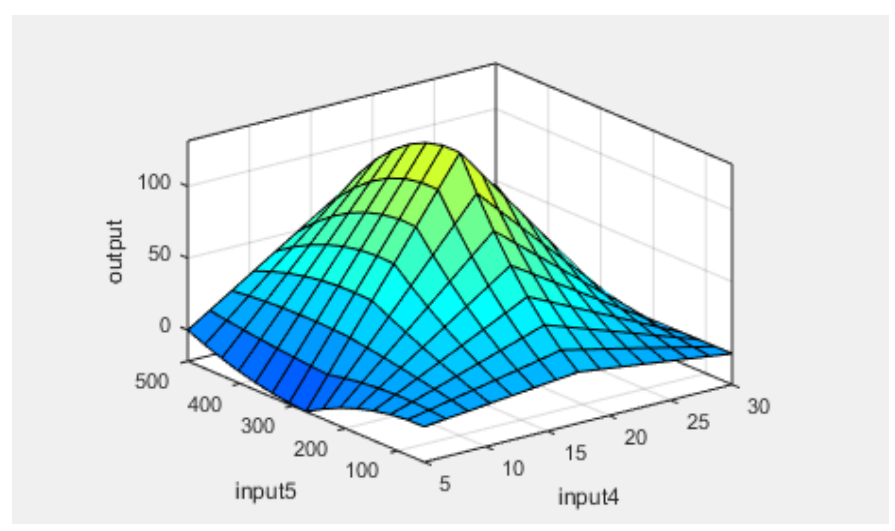

Figure 16. Surfview of output

All the 3D surfviews of the outputs clearly shows that output varies when the input variables are changed. Hence, by the defined ranges of the membership functions, the user category can be depicted by their defined ranges.

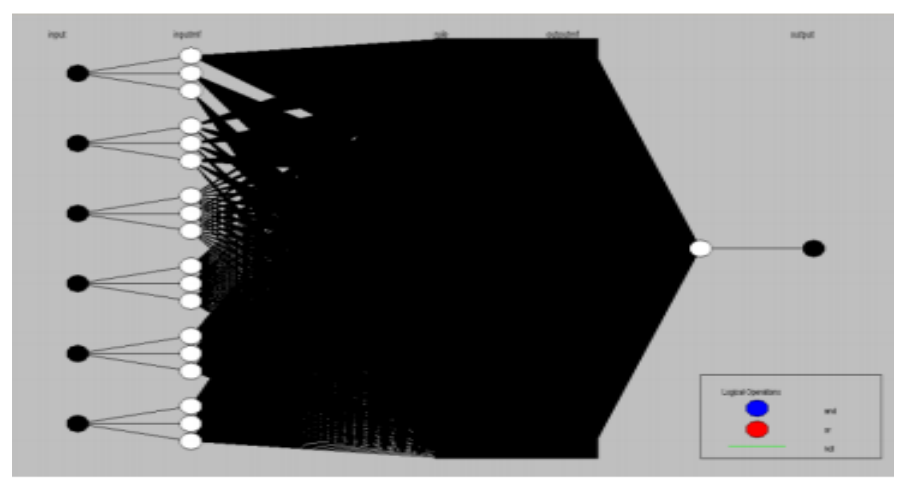

Figure 17. Correlation of inputs with the defined category of OSN user 
After training of the data sets, the final output is obtained which shows the category of user. Multiple scenarios have been taken and discussed above. It takes six input parameters and decides the decision-making behavior of the user. The images show the implementation of this model which tends to detect the decision-making behavior of the online social network user.

\section{Conclusion}

Social-Network has acquired noteworthy consideration as of late, to a great extent because of the achievement of OSN informal and the sites for media-sharing, and the resulting accessibility of an abundance of the data for the social network. Despite the developing interest, yet, there is a small comprehension of the potential business applications of mining social-network. In this era, lots of organizations have realized that if used well, social media can increase their brand awareness and influence the decision-making process of their online community. The making of decisions is ubiquitous across time and space and pervades every facet of our lives. Online Social Networks (OSNs) allow us to find a variety of answers, interpretations, models, and choices [3]. Our research provides a two facet approach, here on process is of mapping the problem qualitatively and determining the relation of decision making with OSN as the DM situations that involve interactions with OSNs which, according to research, are used by $50-60 \%$ of developed countries. A model-based approach that detects the behavior of the consumer and decision-taking style is proposed as its parameters create automation and adaptive process of scrutinizing the type of user by taking certain input values. Hence the results show that these days OSN has a positive impact on user decision making. They are used to develop an opinion, buying, and communication as well and the user can be labeled into categories for ease.

\section{Future Work}

Our research included 146 respondents, the number of respondents can be increased and the more refined decisionmaker can be developed. The fuzzy rule-based can be altered according to the country and region in which the process is being carried out. Moreover, this study will attract various stakeholders to apply certain parameters to the user and detect their kind according to which they can advertise and trigger consumer desire.

\section{References}

[1] Gross, R. and A. Acquisti. Information Revelation And Privacy In Online Social Networks. in Proceedings of the 2005 ACM workshop on Privacy in the electronic society. 2005. ACM.

[2] Jamali, M. and H. Abolhassani. Different Aspects Of Social Network Analysis. in 2006 IEEE/WIC/ACM International Conference on Web Intelligence (WI 2006 Main Conference Proceedings)(WI'06). 2006. IEEE.
[3] Persia, F. and D. D'Auria. A Survey Of Online Social Networks: Challenges And Opportunities. in 2017 IEEE International Conference on Information Reuse and Integration (IRI). 2017. IEEE.

[4] Gupta, M.M. Fuzzy Logic And Fuzzy Systems: Recent Developments And Future Directions. in Proceedings of North American Fuzzy Information Processing. 1996. IEEE.

[5] Qingyang, L., X. Yuxuan, and C. Sijia. Y-Generation Digital Natives' Impulsive Buying Behavior. in 2018 3rd Technology Innovation Management and Engineering Science International Conference (TIMES-iCON). 2018. IEEE.

[6] Gillen, J.J., M. Freeman, and H. Tootell. Human Behavior in Online Social Networks. in IEEE International Symposium on Technology and Society (ISTAS). 2017.

[7] Bilal, G., M.A. Ahmed, and M. Shehzad, Role Of Social Media And Social Networks In Consumer Decision Making: A Case Of The Garment Sector. International Journal of Multidisciplinary Sciences and Engineering, 2014. 5(3): p. $1-9$.

[8] Li, Y.-L., Q.-F. Yan, and J.-W. Wu. Multi-Attribute Group Decision Making Method Based On Evidence Theory. in 2017 13th International Conference on Natural Computation, Fuzzy Systems and Knowledge Discovery (ICNC-FSKD). 2017. IEEE.

[9] Shabbir, A., Shabbir, M., Rizwan, M., \& Ahmad, F. (2019). Ensuring the Confidentiality of Nuclear Information at Cloud Using Modular Encryption Standard. Security and Communication Networks, 2019.

[10] Shozi, N.A. and J. Mtsweni. Big Data Privacy in Social Media Sites. in IST-Africa Week Conference 2017

[11] Jia, Y., et al. Topic Analysis Model for Online Social Network. in 2018 IEEE Third International Conference on Data Science in Cyberspace (DSC). 2018. IEEE.

[12] Sadovykh, V. and D. Sundaram. How Do Online Social Networks Support Decision Making? A Pluralistic Research Agenda. in 2016 49th Hawaii International Conference on System Sciences (HICSS). 2016. IEEE.

[13] Sadovykh, V., D. Sundaram, and S. Piramuthu, Do Online Social Networks Support Decision-Making? Decision support systems, 2015. 70: p. 15-30.

[14] Shahzadi, S., Khaliq, B., Rizwan, M., \& Ahmad, F. (2020). Security of Cloud Computing Using Adaptive Neural Fuzzy Inference System. Security and Communication Networks, 2020.

[15] Akar, E., H.F. Yüksel, and Z.A. Bulut, The Impact Of Social Influence On The Decision-Making Process Of Sports Consumers On Facebook. İnternet Uygulamaları ve Yönetimi Dergisi, 2015. 6(2): p. 5-27. 
[16] Simon, H.A., Theories Of Decision-Making In Economics And Behavioral Science. The American economic review, 1959. 49(3): p. 253-283.

[17] Gulsum Akkuzu, B. Aziz, and M. Adda, Fuzzy Logic Decision based Collaborative Privacy Management Framework for Online Social Networks, in International Conference on Information Systems Security and Privacy. 2019, School of Computing, University of Portsmouth, Portsmouth, U.K.

[18] Cardozo, D.O., M. Pardo, and C.R. Algarín. Fuzzy Logic Controller for Maximum Power Point Tracking Based on Voltage Error Measurement in Isolated Photovoltaic Systems. in 2018 IEEE ANDESCON. 2018. IEEE.

[19] X. Tao, Y. Miao, Y. Zhang, and Z. Shen, "Collaborative medical diagnosis through Fuzzy Petri Net based agent argumentation," IEEE Int. Conf. Fuzzy Syst., no. February, pp. 1197-1204, 2014, doi: 10.1109/FUZZIEEE.2014.6891884.
[20] J. Rong, S. Michalska, S. Subramani, J. Du, and H. Wang, "Deep learning for pollen allergy surveillance from twitter in Australia," BMC Med. Inform. Decis. Mak., vol. 19, no. 1, pp. 1-13, 2019, doi: 10.1186/s12911-019-0921-x.

[21] M. R. Islam, M. A. Kabir, A. Ahmed, A. R. M. Kamal, H. Wang, and A. Ulhaq, "Depression detection from social network data using machine learning techniques," Heal. Inf. Sci. Syst., vol. 6, no. 1, pp. 0-12, 2018, doi: 10.1007/s13755018-0046-0.

[22] Y. Miao, "Modelling dynamic causal relationship in fuzzy cognitive maps," IEEE Int. Conf. Fuzzy Syst., pp. 10131020, 2014, doi: 10.1109/FUZZ-IEEE.2014.6891648.

[23] Y. Miao, C. Miao, X. Tao, Z. Shen, and Z. Liu, "Transformation of cognitive maps," IEEE Trans. Fuzzy Syst., vol. 18, no. 1, pp. 114-124, 2010, doi: 10.1109/TFUZZ.2009.2037218. 